\title{
A Large Nuclear Gravitational Constant and its Universal Applications
}

\author{
Seshavatharam.U.V.S ${ }^{1}$ and S. Lakshminarayana ${ }^{2}$ \\ ${ }^{1}$ Honorary faculty, I-SERVE, Survey no-42, Hitech city, Hyderabad-84,Telangana, INDIA \\ ${ }^{2}$ Dept. of Nuclear Physics, Andhra University, Visakhapatnam-03,AP, INDIA \\ emails: seshavatharam.uvs@gmail.com and sln@auvsp.edu.in/lnsrirama@gmail.com
}

\begin{abstract}
With reference to our earlier published views on large nuclear gravitational constant $G_{s}$, nuclear elementary charge $e_{s}$ and strong coupling constant $\alpha_{s} \cong\left(e / e_{s}\right)^{2}$, in this paper, we present simple relations for nuclear stability range, binding energy of isotopes and magic proton numbers. Even though 'speculative' in nature, proposed concepts seem to be: simple to understand, easy to implement, result oriented, effective and unified. Our proposed model seems to span across the Planck scale and nuclear scale and can be called as SPAN model (STRANGE* physics of atomic nucleus).
\end{abstract}

Summary: Probable range of stable mass numbers can be estimated with $A_{s} \cong\left[Z+\sqrt{\left(1 / \alpha_{s}\right)} \pm 1\right]^{x}$ where $x \cong 1.2$ for $\mathrm{Z} \approx(3$ to 100$)$ and $x \cong 1.19$ for $\mathrm{Z} \geq 100 . \quad A_{s}$ can also be expressed as, $A_{s} \cong 2 Z+k Z^{2}$ where $k \cong\left[4 \pi \varepsilon_{0}(\hbar / 2)^{2} m_{e} c^{2} / e^{2} G_{s} m_{p}^{3}\right] \cong 0.006333$. Energy coefficient being $\left[e_{s}^{2} / 8 \pi \varepsilon_{0}\left(G_{s} m_{p} / c^{2}\right)\right] \approx 10.06 \mathrm{MeV}$, for $Z \approx(5$ to 118$)$, nuclear binding energy can be understood/fitted with two terms as, $B_{A_{s}} \cong\left\{A_{s}-\left[\left(k A_{s} Z / 2.531\right)+3.531\right]\right\} \times 10.06 \mathrm{MeV}$ where $\ln (1 / \sqrt{k}) \cong\left(m_{n}-m_{p} / m_{e}\right) \cong 2.531$. By considering a third term of the form $\left[\left(A_{s}-A\right)^{2} / A_{s}\right]$, binding energy of isotopes of $Z$ can be fitted approximately. It needs further investigation. See section-12 for an in depth discussion.

Keywords: Strong nuclear gravity, nuclear elementary charge, strong coupling constant, nuclear stability range, binding energy of isotopes, magic proton numbers.

\section{Introduction}

With reference to the figure of 'Strong (nuclear) gravity' [1-20], if $G_{f} \approx 10^{38} G_{N}$ and with reference to our recent symposium proceedings and journal publications [21-37], we try to refine our proposed concepts with the following three assumptions for a better understanding on nuclear stability range, binding energy of isotopes and magic proton numbers. $\mathrm{We}$ consider, $G_{f} \cong G_{s} \cong \frac{4 \pi \varepsilon_{0} h^{2} c^{2} m_{e}}{e^{2} m_{p}^{3}} \cong 3.329561 \times 10^{28} \mathrm{~m}^{3} \mathrm{~kg}^{-1} \mathrm{sec}^{-2}$.

\section{Three simple assumptions}

1) Nuclear charge radius can be addressed with, $R_{0} \cong \frac{2 G_{s} m_{p}}{c^{2}} \cong 1.23929083 \mathrm{fm}$

2) Strong coupling constant can be expressed with, $\alpha_{s} \cong\left(\frac{\hbar c}{G_{s} m_{p}^{2}}\right)^{2} \cong 0.1151937353$
3) There exists a nuclear elementary charge, $e_{s} \cong \frac{e}{\sqrt{\alpha_{s}}} \cong\left(\frac{G_{s} m_{p}^{2}}{\hbar c}\right) e \cong 4.720586027 \times 10^{-19} \mathrm{C}$

\section{Semi empirical relations and applications}

1) Fine structure ratio can be addressed with, $\alpha \cong \frac{2 \pi h c m_{e}}{G_{s} m_{p}^{3}} \cong \frac{4 \pi^{2} \hbar c m_{e}}{G_{s} m_{p}^{3}} \cong\left(\frac{e_{s}^{2}}{4 \pi \varepsilon_{0} G_{s} m_{p}^{2}}\right)\left(\frac{\hbar c}{G_{s} m_{p}^{2}}\right)$

2) With reference to Newtonian gravitational constant $G_{N}$, proton-electron mass ratio can be addressed with (See section-12, point no.18),

$$
\frac{m_{p}}{m_{e}} \cong\left(\frac{e_{s} G_{s}}{e G_{N}}\right)^{\frac{1}{12}} \cong\left(\frac{G_{s}^{2} m_{p}^{2}}{G_{N} \hbar c}\right)^{\frac{1}{12}}
$$

3) Proton magnetic moment can be addressed with $\mu_{p} \cong \frac{e_{s} \hbar}{2 m_{p}} \cong \frac{e G_{s} m_{p}}{2 c} \cong 1.488142 \times 10^{-26}{\mathrm{~J} . \mathrm{T}^{-1}}^{-1}$

4) Neutron magnetic moment can be addressed with $\mu_{n} \cong \frac{\left(e_{s}-e\right) \hbar}{2 m_{n}} \cong 9.817102 \times 10^{-27}{\mathrm{~J} . \mathrm{T}^{-1} .}^{-}$

*STRANGE: Salam/Sinha/Sivaram/Sabbatta, Tennakone, Rutherford, Avagodaro, Newton, Gamow, Einstein etc. 
5) Nuclear unit radius can be expressed as, $R_{0} \cong \frac{2 G_{s} m_{p}}{c^{2}} \cong\left(\frac{e}{e}\right)\left\{\frac{\hbar}{m_{p} c}+\frac{\hbar}{m_{n} c}\right\}$

6) Root mean square nuclear charge radii can be addressed with,

$R_{(Z, A)} \cong\left\{Z^{1 / 3}+(\sqrt{\mathrm{Z}(A-Z)})^{1 / 3}\right\}\left(\frac{G_{s} m_{p}}{c^{2}}\right)$

7) Nuclear potential energy can be understood with, $\cong \frac{e_{s}^{2}}{4 \pi \varepsilon_{0}\left(G_{s} m_{p} / c^{2}\right)} \cong 20.1734 \mathrm{MeV}$

8) Nuclear binding energy can be understood with , $\frac{e^{2} G_{s} m_{p}^{3}}{8 \pi \varepsilon_{0} \hbar^{2}} \cong \frac{e_{s} e}{8 \pi \varepsilon_{0}\left(\hbar / m_{p} c\right)} \cong \frac{e_{s}^{2}}{8 \pi \varepsilon_{0}\left(G_{s} m_{p} / c^{2}\right)}$ $\cong 10.0867 \mathrm{MeV}$

9) With reference to $(\hbar / 2)$, a useful quantum energy constant can be expressed with, $E_{(\hbar / 2)} \cong\left(\frac{e^{2} G_{s} m_{p}^{3}}{4 \pi \varepsilon_{0}(\hbar / 2)^{2}}\right) \cong 80.6934 \mathrm{MeV}$

10) Close to magic and semi magic proton numbers, nuclear binding energy seems to approach $\left[2.531\left(n+\frac{1}{2}\right)\right]^{2} 10.0 \mathrm{MeV}$ where $\mathrm{n}=0,1,2,3, \ldots$ and $\left(m_{n}-m_{p} / m_{e}\right)=2.531$.

11) Characteristic melting temperature associated with proton can be expressed with, $T_{\text {proton }} \cong \frac{\hbar c^{3}}{8 \pi k_{B} G_{s} m_{p}} \cong 0.15 \times 10^{12} \mathrm{~K}$

12) Characteristic nuclear neutral mass unit [33] can be addressed with, $\sqrt{\frac{\hbar c}{G_{s}}} \cong 546.62 \mathrm{MeV} / c^{2}$. It can also be considered as a characteristic dark matter constituent [12]. See relation (26) and table- 8 of section -12 for the estimated basic baryonic mass spectrum.

\section{To fit neutron-proton mass difference}

Neutron-proton mass difference can be understood with:

$$
\left(\frac{m_{n} c^{2}-m_{p} c^{2}}{m_{e} c^{2}}\right) \cong \ln \sqrt{\frac{E_{(\hbar / 2)}}{m_{e} c^{2}}} \cong \ln \sqrt{\frac{4 e^{2} G_{s} m_{p}^{3}}{4 \pi \varepsilon_{0} \hbar^{2} m_{e} c^{2}}}
$$

\section{To fit neutron life time}

Neutron life time $t_{n}$ can be understood with the following relation:

$$
t_{n} \cong \exp \left(\frac{E_{(\hbar / 2)}}{\left(m_{n}-m_{p}\right) c^{2}}\right) \times\left(\frac{\hbar}{m_{n} c^{2}}\right) \cong 877.3 \mathrm{sec}
$$

This value can be compared with recommended value of $(878.5 \pm 0.8) \mathrm{sec}$.

\section{Understanding proton-neutron stability}

$$
\text { Let, }\left(\frac{m_{e} c^{2}}{E_{(\hbar / 2)}}\right) \cong\left(\frac{4 \pi \varepsilon_{0} \hbar^{2} m_{e} c^{2}}{4 e^{2} G_{s} m_{p}^{3}}\right) \cong k \cong 0.0063326
$$

Quantitatively, we noticed that,

$$
\left.\begin{array}{l}
\frac{e_{s}^{2}}{4 \pi \varepsilon_{0} G_{s} m_{p} m_{e}} \cong 4 \pi^{2} \cong \frac{1}{4 k} \\
\exp \left(\frac{m_{n}-m_{p}}{m_{e}}\right) \cong 4 \pi \cong \sqrt{\frac{1}{k}}
\end{array}\right\}
$$

The new factor $k$ needs a clear interpretation and we are working on that for its scope and applicability. It can be considered as a result oriented number connected with nuclear stability and binding energy.

Stable mass number $A_{s}$ of $Z$ can be estimated with the following simple relations [38],

$$
\begin{gathered}
A_{s} \cong\left(N_{s}+Z\right) \cong 2 Z+k Z^{2} \cong 2 Z+0.0063326(Z)^{2} \\
A_{s} \cong\left[Z+\sqrt{\left(1 / \alpha_{s}\right)}\right]^{1.2} \cong[Z+2.9463]^{1.2}
\end{gathered}
$$

where $\left(e / e_{s}\right)(1 / k)^{1 / 4} \cong\left(\alpha_{s}\right)^{1 / 2}(1 / k)^{1 / 4} \cong 1$. . It can be called as 'power factor of stability'.

Proton number $Z$ associated with stable $A_{s}$ can be estimated with the following simple relations,

$$
Z \cong \frac{\sqrt{1+k A_{s}}-1}{k} \text { Or } Z \cong \frac{A_{s}}{1+\sqrt{1+k A_{s}}}
$$

\section{Understanding proton-neutron stability range}

Considering relation (8), it seems possible to find the best possible range of $A_{s}$. We noticed that,

$$
\left.\begin{array}{l}
\left(A_{s}\right)_{\text {mean }} \cong\left[Z+\sqrt{\left(1 / \alpha_{s}\right)}\right]^{1.2} \\
\left(A_{s}\right)_{\text {low }}^{\text {up }} \cong\left[Z+\left(\sqrt{\left(1 / \alpha_{s}\right)} \pm 1\right)\right]^{1.2}
\end{array}\right\}
$$


A large nuclear gravitational constant and its universal applications

Lower stable $A_{s}$ can be estimated with,

$$
\left(A_{s}\right)_{\text {low }} \cong\left[Z+\left(\sqrt{\left(1 / \alpha_{s}\right)}-1\right)\right]^{1.2} \cong[Z+1.9463]^{1.2}
$$

Upper stable $A_{s}$ can be estimated with,

$$
\left(A_{s}\right)_{u p} \cong\left[Z+\left(\sqrt{\left(1 / \alpha_{s}\right)}+1\right)\right]^{1.2} \cong[Z+3.9463]^{1.2}
$$

See table-1 for the estimated range of stable mass numbers for $\mathrm{Z}=3$ to 100 . With even-odd corrections data can be refined.

Considering a factor of 1.19 in place of 1.2 , stable mass numbers of super heavy elements can be fitted. For $\mathrm{Z}=116$, estimated stable mass number range seems to be 292 to 298 and its experimental mass range is 291 to 294 [39]. See table 2 for a comparison.

Table-1: Estimated range of stable mass numbers

\begin{tabular}{|c|c|c|c|c|}
\hline$Z$ & $\left(A_{s}\right)_{l o w}$ & $\left(A_{s}\right)_{\text {mean }}$ & $\left(A_{s}\right)_{u p}$ & $\begin{array}{l}\text { Main } \\
\text { Isotope } \\
\text { range }\end{array}$ \\
\hline 3 & 7 & 8 & 10 & 6 to 7 \\
\hline 4 & 8 & 10 & 12 & 7 to 10 \\
\hline 5 & 10 & 12 & 14 & 10 to 11 \\
\hline 6 & 12 & 14 & 16 & 11 to 14 \\
\hline 7 & 14 & 16 & 18 & 13 to 15 \\
\hline 8 & 16 & 18 & 20 & 16 to 18 \\
\hline 9 & 18 & 20 & 22 & 18 to 19 \\
\hline 10 & 20 & 22 & 24 & 20 to 22 \\
\hline 11 & 22 & 24 & 26 & 22 to 24 \\
\hline 12 & 24 & 26 & 28 & 24 to 26 \\
\hline 13 & 26 & 28 & 30 & 26 to 27 \\
\hline 14 & 28 & 30 & 32 & 28 to 32 \\
\hline 15 & 30 & 32 & 34 & 31 to 33 \\
\hline 16 & 32 & 34 & 36 & 32 to 36 \\
\hline 17 & 34 & 36 & 38 & 35 to 37 \\
\hline 18 & 36 & 38 & 41 & 36 to 42 \\
\hline 19 & 38 & 41 & 43 & 39 to 41 \\
\hline 20 & 41 & 43 & 45 & 40 to 48 \\
\hline 21 & 43 & 45 & 47 & 44 to 48 \\
\hline 22 & 45 & 47 & 50 & 46 to 50 \\
\hline 23 & 47 & 50 & 52 & 48 to 51 \\
\hline 24 & 50 & 52 & 54 & 50 to 54 \\
\hline 25 & 52 & 54 & 57 & 52 to 55 \\
\hline 26 & 54 & 57 & 59 & 54 to 60 \\
\hline 27 & 57 & 59 & 61 & 56 to 60 \\
\hline 28 & 59 & 61 & 64 & 58 to 64 \\
\hline 29 & 61 & 64 & 66 & 63 to 67 \\
\hline 30 & 64 & 66 & 69 & 64 to 72 \\
\hline 31 & 66 & 69 & 71 & 66 to 73 \\
\hline 32 & 69 & 71 & 74 & 68 to 76 \\
\hline 33 & 71 & 74 & 76 & 73 to 75 \\
\hline 34 & 74 & 76 & 79 & 72 to 82 \\
\hline 35 & 76 & 79 & 81 & 79,81 \\
\hline 36 & 79 & 81 & 84 & 78 to 86 \\
\hline 37 & 81 & 84 & 86 & 83 to 87 \\
\hline 38 & 84 & 86 & 89 & 82 to 88 \\
\hline 39 & 86 & 89 & 91 & 87 to 91 \\
\hline 40 & 89 & 91 & 94 & 88 to 96 \\
\hline
\end{tabular}
for $Z=3$ to 100 with a power factor of 1.20

\begin{tabular}{|c|c|c|c|c|}
\hline 41 & 91 & 94 & 96 & 90 to 96 \\
\hline 42 & 94 & 96 & 99 & 92 to 100 \\
\hline 43 & 96 & 99 & 101 & 95 to 99 \\
\hline 44 & 99 & 101 & 104 & 96 to 106 \\
\hline 45 & 101 & 104 & 107 & 99 to 105 \\
\hline 46 & 104 & 107 & 109 & 100 to 110 \\
\hline 47 & 107 & 109 & 112 & 105 to 111 \\
\hline 48 & 109 & 112 & 114 & 106 to 116 \\
\hline 49 & 112 & 114 & 117 & 113,115 \\
\hline 50 & 114 & 117 & 120 & 112 to 126 \\
\hline 51 & 117 & 120 & 122 & 121 to 125 \\
\hline 52 & 120 & 122 & 125 & 120 to 130 \\
\hline 53 & 122 & 125 & 128 & 123 to 135 \\
\hline 54 & 125 & 128 & 131 & 124 to 136 \\
\hline 55 & 128 & 131 & 133 & 133 to 137 \\
\hline 56 & 131 & 133 & 136 & 130 to 138 \\
\hline 57 & 133 & 136 & 139 & 137 to 139 \\
\hline 58 & 136 & 139 & 141 & 134 to 144 \\
\hline 59 & 139 & 141 & 144 & 141 to 143 \\
\hline 60 & 141 & 144 & 147 & 142 to 150 \\
\hline 61 & 144 & 147 & 150 & 145 to 147 \\
\hline 62 & 147 & 150 & 152 & 144 to 154 \\
\hline 63 & 150 & 152 & 155 & 150 to 155 \\
\hline 64 & 152 & 155 & 158 & 148 to 160 \\
\hline 65 & 155 & 158 & 161 & 157 to 159 \\
\hline 66 & 158 & 161 & 164 & 154 to 164 \\
\hline 67 & 161 & 164 & 166 & 163 to 167 \\
\hline 68 & 164 & 166 & 169 & 160 to 172 \\
\hline 69 & 166 & 169 & 172 & 167 to 171 \\
\hline 70 & 169 & 172 & 175 & 166 to 177 \\
\hline 71 & 172 & 175 & 178 & 173 to 176 \\
\hline 72 & 175 & 178 & 181 & 172 to 182 \\
\hline 73 & 178 & 181 & 183 & 177 to 183 \\
\hline 74 & 181 & 183 & 186 & 180 to 186 \\
\hline 75 & 183 & 186 & 189 & 185,187 \\
\hline 76 & 186 & 189 & 192 & 184 to 194 \\
\hline 77 & 189 & 192 & 195 & 188 to 194 \\
\hline 78 & 192 & 195 & 198 & 190 to 198 \\
\hline 79 & 195 & 198 & 201 & 195 to 199 \\
\hline 80 & 198 & 201 & 204 & 194 to 204 \\
\hline 81 & 201 & 204 & 207 & 203 to 205 \\
\hline 82 & 204 & 207 & 209 & 202 to 214 \\
\hline 83 & 207 & 209 & 212 & 207 to 210 \\
\hline 84 & 209 & 212 & 215 & 208 to 210 \\
\hline 85 & 212 & 215 & 218 & 209 to 211 \\
\hline 86 & 215 & 218 & 221 & 218 to 224 \\
\hline 87 & 218 & 221 & 224 & 221 to 223 \\
\hline 88 & 221 & 224 & 227 & 223 to 228 \\
\hline 89 & 224 & 227 & 230 & 225 to 227 \\
\hline 90 & 227 & 230 & 233 & 227 to 234 \\
\hline 91 & 230 & 233 & 236 & 229 to 234 \\
\hline 92 & 233 & 236 & 239 & 232 to 238 \\
\hline 93 & 236 & 239 & 242 & 235 to 239 \\
\hline 94 & 239 & 242 & 245 & 238 to 244 \\
\hline 95 & 242 & 245 & 248 & 241 to 243 \\
\hline 96 & 245 & 248 & 251 & 242 to 250 \\
\hline 97 & 248 & 251 & 254 & 245 to 249 \\
\hline 98 & 251 & 254 & 257 & 248 to 254 \\
\hline 99 & 254 & 257 & 260 & 252 to 255 \\
\hline 100 & 257 & 260 & 263 & 252 to 257 \\
\hline
\end{tabular}


A large nuclear gravitational constant and its universal applications

Table-2: Estimated range of stable mass numbers for $Z=101$ to 118 with a power factor of 1.19

\begin{tabular}{|c|c|c|c|c|}
\hline$Z$ & $\left(A_{s}\right)_{\text {low }}$ & $\left(A_{s}\right)_{\text {mean }}$ & $\left(A_{s}\right)_{\text {up }}$ & $\begin{array}{c}\text { Current } \\
\text { synthetic } \\
\text { isotopes range }\end{array}$ \\
\hline 101 & 248 & 251 & 254 & 257 to 260 \\
\hline 102 & 251 & 254 & 257 & 253 to 259 \\
\hline 103 & 254 & 257 & 260 & 254 to 266 \\
\hline 104 & 257 & 260 & 263 & 261 to 267 \\
\hline 105 & 260 & 263 & 266 & 262 to 270 \\
\hline 106 & 263 & 266 & 269 & 265 to 271 \\
\hline 107 & 266 & 269 & 271 & 267 to 278 \\
\hline 108 & 269 & 271 & 274 & 269 to 271 \\
\hline 109 & 271 & 274 & 277 & 274 to 282 \\
\hline 110 & 274 & 277 & 280 & 279 to 281 \\
\hline 111 & 277 & 280 & 283 & 279 to 286 \\
\hline 112 & 280 & 283 & 286 & 277 to 285 \\
\hline 113 & 283 & 286 & 289 & 278 to 290 \\
\hline 114 & 286 & 289 & 292 & 284 to 290 \\
\hline 115 & 289 & 292 & 295 & 287 to 290 \\
\hline 116 & 292 & 295 & 298 & 290 to 294 \\
\hline 117 & 295 & 298 & 301 & 293,294 \\
\hline 118 & 298 & 301 & 304 & 294,295 \\
\hline
\end{tabular}

8. Nuclear binding energy close to stable mass numbers

Based on the new integrated model proposed by N. Ghahramany et al $[40,41]$,

$$
B(Z, N)=\left\{A-\left(\frac{\left(N^{2}-Z^{2}\right)+\delta(N-Z)}{3 Z}+3\right)\right\} \frac{m_{n} c^{2}}{\gamma}
$$

where, $\gamma=$ Adjusting coefficient $\approx(90$ to 100$)$.

if $N \neq Z, \delta(N-Z)=0$ and if $N=Z, \delta(N-Z)=1$.

Readers are encouraged to see references there in $[40,41]$ for derivation part. Point to be noted is that, close to the beta stability line, $\left[\frac{N^{2}-Z^{2}}{3 Z}\right]$ takes care of the combined effects of coulombic and asymmetric effects. In this context, we would like suggest that,

$$
\left.\begin{array}{l}
\frac{m_{n} c^{2}}{\gamma} \cong \frac{m_{n} c^{2}}{(90 \text { to } 100)} \cong \text { Constant } \\
\cong \frac{e_{s}^{2}}{8 \pi \varepsilon_{0}\left(G_{s} m_{p} / c^{2}\right)} \cong 10.09 \mathrm{MeV}
\end{array}\right\}
$$

Proceeding further, with reference to relation (7), it is also possible to show that, for $Z \cong(40$ to 83$)$, close to the beta stability line,

$$
\begin{gathered}
{\left[\frac{N_{s}^{2}-Z^{2}}{Z}\right] \cong k A_{s} Z} \\
{\left[\frac{N_{s}^{2}-Z^{2}}{3 Z}\right] \cong \frac{k A_{s} Z}{3}}
\end{gathered}
$$

Based on the above relations and close to the stable mass numbers of $(\mathrm{Z} \approx 5$ to 118$)$, with a common energy coefficient of $10.06 \mathrm{MeV}$, we would like to suggest two terms for fitting and understanding nuclear binding energy.

First term helps in increasing the binding energy and can be considered as,

$$
\text { Term } \_1=A_{s} \times 10.06 \mathrm{MeV}
$$

Second term helps in decreasing the binding energy and can be considered as,

$$
\text { Term_2 }=\left(\frac{k A_{s} Z}{2.531}+3.531\right) \times 10.06 \mathrm{MeV}
$$

$$
\text { where }\left\{\begin{array}{l}
\left(\frac{\left(m_{n}-m_{p}\right) c^{2}}{m_{e} c^{2}}\right) \cong \ln \left(\frac{1}{\sqrt{k}}\right) \cong 2.531 \\
3.531 \cong 1+2.531 \cong 1+\ln \left(\frac{1}{\sqrt{k}}\right)
\end{array}\right.
$$

Thus, binding energy can be fitted with,

$$
B_{A_{s}} \cong\left\{A_{s}-\left(\frac{k A_{s} Z}{2.531}+3.531\right)\right\} \times 10.06 \mathrm{MeV}
$$

See the following figure 1 . Dotted red curve plotted with relations (7) and (20) can be compared with the green curve plotted with the standard semi empirical mass formula (SEMF) [38,42].

Figure 1: Binding energy per nucleon close to stable mass numbers of $Z=5$ to 118

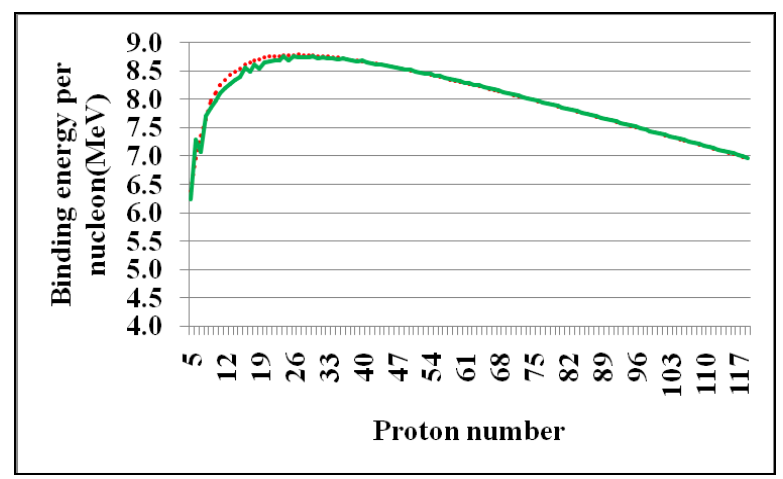


A large nuclear gravitational constant and its universal applications

For medium and heavy atomic nuclides, fit is excellent. It seems that some correction is required for light atoms. See table 3 for the estimated data.

Table 3: Nuclear Binding energy close to stable mass numbers of $Z=5$ to 118

\begin{tabular}{|c|c|c|c|c|}
\hline $\begin{array}{l}\text { Proton } \\
\text { number }\end{array}$ & $\begin{array}{c}\text { Mass } \\
\text { number }\end{array}$ & $\begin{array}{c}\text { Estd. BE } \\
(\mathrm{MeV})\end{array}$ & $\begin{array}{c}\text { SEMF } \\
\text { BE } \\
(\mathrm{MeV}) \\
\end{array}$ & $\begin{array}{c}\text { Error } \\
(\mathrm{MeV})\end{array}$ \\
\hline 5 & 10 & 63.8 & 62.3 & -1.53 \\
\hline 6 & 12 & 83.4 & 87.4 & 4.01 \\
\hline 7 & 14 & 102.9 & 98.8 & -4.04 \\
\hline 8 & 16 & 122.2 & 123.2 & 1.03 \\
\hline 9 & 19 & 151.3 & 148.9 & -2.46 \\
\hline 10 & 21 & 170.5 & 167.5 & -2.94 \\
\hline 11 & 23 & 189.5 & 186.1 & -3.35 \\
\hline 12 & 25 & 208.4 & 204.7 & -3.71 \\
\hline 13 & 27 & 227.3 & 223.2 & -4.04 \\
\hline 14 & 29 & 246.0 & 241.6 & -4.35 \\
\hline 15 & 31 & 264.6 & 260.0 & -4.65 \\
\hline 16 & 34 & 292.8 & 290.8 & -2.06 \\
\hline 17 & 36 & 311.2 & 305.1 & -6.18 \\
\hline 18 & 38 & 329.5 & 327.2 & -2.32 \\
\hline 19 & 40 & 347.7 & 341.5 & -6.27 \\
\hline 20 & 43 & 375.4 & 371.6 & -3.84 \\
\hline 21 & 45 & 393.4 & 389.6 & -3.80 \\
\hline 22 & 47 & 411.3 & 407.5 & -3.80 \\
\hline 23 & 49 & 429.1 & 425.2 & -3.85 \\
\hline 24 & 52 & 456.2 & 454.6 & -1.61 \\
\hline 25 & 54 & 473.7 & 468.9 & -4.85 \\
\hline 26 & 56 & 491.2 & 489.6 & -1.61 \\
\hline 27 & 59 & 517.9 & 515.2 & -2.72 \\
\hline 28 & 61 & 535.1 & 532.5 & -2.63 \\
\hline 29 & 63 & 552.3 & 549.7 & -2.61 \\
\hline 30 & 66 & 578.6 & 577.9 & -0.67 \\
\hline 31 & 68 & 595.5 & 592.0 & -3.52 \\
\hline 32 & 70 & 612.3 & 611.7 & -0.60 \\
\hline 33 & 73 & 638.2 & 636.6 & -1.60 \\
\hline 34 & 75 & 654.8 & 653.3 & -1.52 \\
\hline 35 & 78 & 680.4 & 677.9 & -2.56 \\
\hline 36 & 80 & 696.8 & 697.0 & 0.26 \\
\hline 37 & 83 & 722.2 & 721.3 & -0.84 \\
\hline 38 & 85 & 738.3 & 737.6 & -0.69 \\
\hline 39 & 88 & 763.4 & 761.6 & -1.80 \\
\hline 40 & 90 & 779.3 & 780.2 & 0.93 \\
\hline 41 & 93 & 804.1 & 803.9 & -0.21 \\
\hline 42 & 95 & 819.7 & 819.7 & 0.00 \\
\hline 43 & 98 & 844.3 & 843.2 & -1.13 \\
\hline 44 & 100 & 859.7 & 861.2 & 1.52 \\
\hline 45 & 103 & 884.0 & 884.4 & 0.38 \\
\hline 46 & 105 & 899.2 & 899.8 & 0.62 \\
\hline 47 & 108 & 923.2 & 922.7 & -0.49 \\
\hline 48 & 111 & 947.0 & 947.6 & 0.62 \\
\hline 49 & 113 & 961.9 & 962.8 & 0.96 \\
\hline 50 & 116 & 985.5 & 987.5 & 2.03 \\
\hline 51 & 118 & 1000.1 & 1000.2 & 0.16 \\
\hline 52 & 121 & 1023.4 & 1024.6 & 1.22 \\
\hline 53 & 124 & 1046.5 & 1046.5 & 0.05 \\
\hline 54 & 126 & 1060.8 & 1063.4 & 2.62 \\
\hline 55 & 129 & 1083.6 & 1085.1 & 1.47 \\
\hline 56 & 132 & 1106.3 & 1108.7 & 2.38 \\
\hline
\end{tabular}

\begin{tabular}{|c|c|c|c|c|}
\hline 57 & 135 & 1128.9 & 1130.1 & 1.17 \\
\hline 58 & 137 & 1142.7 & 1144.4 & 1.73 \\
\hline 59 & 140 & 1165.0 & 1165.6 & 0.58 \\
\hline 60 & 143 & 1187.1 & 1188.5 & 1.42 \\
\hline 61 & 146 & 1209.1 & 1209.3 & 0.23 \\
\hline 62 & 148 & 1222.4 & 1225.3 & 2.91 \\
\hline 63 & 151 & 1244.1 & 1245.9 & 1.77 \\
\hline 64 & 154 & 1265.6 & 1268.2 & 2.56 \\
\hline 65 & 157 & 1287.0 & 1288.4 & 1.41 \\
\hline 66 & 160 & 1308.3 & 1310.4 & 2.16 \\
\hline 67 & 162 & 1321.0 & 1322.1 & 1.14 \\
\hline 68 & 165 & 1342.0 & 1343.9 & 1.94 \\
\hline 69 & 168 & 1362.8 & 1363.6 & 0.86 \\
\hline 70 & 171 & 1383.4 & 1385.1 & 1.64 \\
\hline 71 & 174 & 1404.0 & 1404.5 & 0.58 \\
\hline 72 & 177 & 1424.3 & 1425.7 & 1.34 \\
\hline 73 & 180 & 1444.5 & 1444.8 & 0.30 \\
\hline 74 & 183 & 1464.6 & 1465.7 & 1.06 \\
\hline 75 & 186 & 1484.5 & 1484.6 & 0.06 \\
\hline 76 & 189 & 1504.3 & 1505.1 & 0.82 \\
\hline 77 & 192 & 1523.9 & 1523.7 & -0.14 \\
\hline 78 & 195 & 1543.3 & 1544.0 & 0.64 \\
\hline 79 & 198 & 1562.6 & 1562.4 & -0.27 \\
\hline 80 & 201 & 1581.8 & 1582.3 & 0.54 \\
\hline 81 & 204 & 1600.8 & 1600.5 & -0.33 \\
\hline 82 & 207 & 1619.7 & 1620.2 & 0.51 \\
\hline 83 & 210 & 1638.4 & 1638.1 & -0.29 \\
\hline 84 & 213 & 1656.9 & 1657.5 & 0.58 \\
\hline 85 & 216 & 1675.3 & 1675.2 & -0.16 \\
\hline 86 & 219 & 1693.6 & 1694.3 & 0.76 \\
\hline 87 & 222 & 1711.7 & 1711.7 & 0.08 \\
\hline 88 & 225 & 1729.6 & 1730.7 & 1.05 \\
\hline 89 & 228 & 1747.4 & 1747.8 & 0.44 \\
\hline 90 & 231 & 1765.0 & 1766.5 & 1.46 \\
\hline 91 & 234 & 1782.5 & 1783.5 & 0.93 \\
\hline 92 & 238 & 1807.6 & 1808.5 & 0.90 \\
\hline 93 & 241 & 1824.8 & 1825.2 & 0.44 \\
\hline 94 & 244 & 1841.8 & 1843.4 & 1.56 \\
\hline 95 & 247 & 1858.7 & 1859.9 & 1.17 \\
\hline 96 & 250 & 1875.4 & 1877.7 & 2.36 \\
\hline 97 & 254 & 1899.6 & 1898.9 & -0.71 \\
\hline 98 & 257 & 1916.0 & 1916.5 & 0.54 \\
\hline 99 & 260 & 1932.2 & 1932.5 & 0.34 \\
\hline 100 & 263 & 1948.3 & 1949.9 & 1.66 \\
\hline 101 & 267 & 1971.7 & 1971.9 & 0.16 \\
\hline 102 & 270 & 1987.5 & 1989.1 & 1.56 \\
\hline 103 & 273 & 2003.1 & 2004.6 & 1.54 \\
\hline 104 & 276 & 2018.6 & 2021.6 & 3.02 \\
\hline 105 & 280 & 2041.3 & 2041.4 & 0.17 \\
\hline 106 & 283 & 2056.4 & 2058.1 & 1.74 \\
\hline 107 & 287 & 2078.7 & 2079.1 & 0.36 \\
\hline 108 & 290 & 2093.5 & 2095.6 & 2.01 \\
\hline 109 & 293 & 2108.2 & 2110.5 & 2.30 \\
\hline 110 & 297 & 2130.0 & 2131.0 & 1.01 \\
\hline 111 & 300 & 2144.3 & 2145.7 & 1.42 \\
\hline 112 & 303 & 2158.5 & 2161.7 & 3.26 \\
\hline 113 & 307 & 2179.7 & 2181.8 & 2.08 \\
\hline 114 & 310 & 2193.6 & 2197.6 & 4.02 \\
\hline 115 & 314 & 2214.4 & 2216.0 & 1.53 \\
\hline 116 & 317 & 2227.9 & 2231.5 & 3.59 \\
\hline 117 & 321 & 2248.4 & 2250.9 & 2.53 \\
\hline 118 & 324 & 2261.6 & 2266.3 & 4.69 \\
\hline
\end{tabular}


A large nuclear gravitational constant and its universal applications

\section{Nuclear binding energy of isotopes of $\mathbf{Z}$}

We are working on understanding and estimating the binding energy of mass numbers above and below the stable mass numbers.

With trial and error, we have developed a third term of the form $\left[\frac{\left(A_{s}-A\right)^{2}}{A_{s}}\right] \times 10.06 \mathrm{MeV}$. Using this

term, approximately, it is possible to fit the binding energy of isotopes in following way.

$$
B_{A} \cong\left\{\left[A-\left(\frac{k A Z}{2.531}+3.531\right)\right]-\left[\frac{\left(A_{s}-A\right)^{2}}{A_{s}}\right]\right\} \times 10.06 \mathrm{MeV}
$$

See figure 2 and table 4 for the estimated isotopic binding energy of $Z=50$. Dashed red curve plotted with relations (7) and (21) can be compared with the green curve plotted with total binding energy of Thomas-Fermi model [42].

For $\mathrm{Z}=50$ and $\mathrm{A}=100$ to 130 , with reference to total binding energy of Thomas-Fermi model [42], there is no much more difference in the estimation of binding energy. When $(A>130)$, binding energy seems to be increasing and when $(A>170)$, binding energy seems to be decreasing rapidly. It needs further study and refinement.

See figures 3 to 10 for the estimated isotopic binding energies of $\mathrm{Z}=22,32,42,52,62,72,92$ and 92 .

\section{Figure 2: Binding energy of isotopes of $\mathrm{Z}=50$}

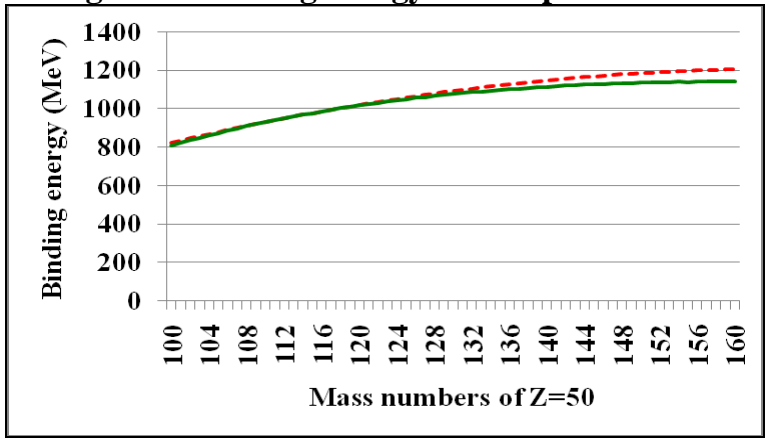

Table 4: Binding energy of isotopes of $Z=50$

\begin{tabular}{|c|c|c|c|c|}
\hline $\begin{array}{c}\text { Proton } \\
\text { number }\end{array}$ & $\begin{array}{c}\text { Mass } \\
\text { number }\end{array}$ & $\begin{array}{c}\text { Estd. } \\
\text { BE } \\
(\mathrm{MeV})\end{array}$ & $\begin{array}{c}\text { Total BE } \\
(\mathrm{MeV}) \\
{[26]}\end{array}$ & $\begin{array}{c}\text { Error } \\
(\mathrm{MeV})\end{array}$ \\
\hline 50 & 100 & 822.4 & 826.0 & 3.6 \\
\hline 50 & 101 & 833.9 & 837.2 & 3.3 \\
\hline 50 & 102 & 845.2 & 850.7 & 5.4 \\
\hline 50 & 103 & 856.4 & 860.7 & 4.3 \\
\hline 50 & 104 & 867.3 & 873.1 & 5.8 \\
\hline 50 & 105 & 878.1 & 882.7 & 4.6 \\
\hline 50 & 106 & 888.8 & 894.6 & 5.8 \\
\hline 50 & 107 & 899.2 & 903.5 & 4.3 \\
\hline
\end{tabular}

\begin{tabular}{|c|c|c|c|c|}
\hline 50 & 108 & 909.5 & 914.9 & 5.5 \\
\hline 50 & 109 & 919.6 & 923.5 & 3.9 \\
\hline 50 & 110 & 929.5 & 934.7 & 5.2 \\
\hline 50 & 111 & 939.3 & 942.9 & 3.6 \\
\hline 50 & 112 & 948.9 & 953.5 & 4.6 \\
\hline 50 & 113 & 958.3 & 961.1 & 2.8 \\
\hline 50 & 114 & 967.5 & 971.4 & 3.9 \\
\hline 50 & 115 & 976.6 & 978.7 & 2.2 \\
\hline 50 & 116 & 985.5 & 988.5 & 3.0 \\
\hline 50 & 117 & 994.2 & 995.4 & 1.3 \\
\hline 50 & 118 & 1002.7 & 1004.7 & 2.0 \\
\hline 50 & 119 & 1011.1 & 1011.3 & 0.2 \\
\hline 50 & 120 & 1019.3 & 1020.3 & 1.1 \\
\hline 50 & 121 & 1027.3 & 1026.8 & -0.5 \\
\hline 50 & 122 & 1035.1 & 1035.5 & 0.4 \\
\hline 50 & 123 & 1042.8 & 1041.5 & -1.3 \\
\hline 50 & 124 & 1050.3 & 1050.1 & -0.2 \\
\hline 50 & 125 & 1057.6 & 1055.8 & -1.8 \\
\hline 50 & 126 & 1064.8 & 1064.1 & -0.7 \\
\hline 50 & 127 & 1071.8 & 1069.6 & -2.2 \\
\hline 50 & 128 & 1078.6 & 1077.5 & -1.0 \\
\hline 50 & 129 & 1085.2 & 1082.8 & -2.4 \\
\hline 50 & 130 & 1091.7 & 1090.5 & -1.2 \\
\hline 50 & 131 & 1098.0 & 1095.61 & -2.3 \\
\hline 50 & 132 & 1104.1 & 1102.6 & -1.5 \\
\hline 50 & 133 & 1110.0 & 1105.2 & -4.8 \\
\hline 50 & 134 & 1115.8 & 1109.5 & -6.2 \\
\hline 50 & 135 & 1121.4 & 1111.4 & -9.9 \\
\hline 50 & 136 & 1126.8 & 1115.2 & -11.6 \\
\hline 50 & 137 & 1132.0 & 1116.9 & -15.1 \\
\hline 50 & 138 & 1137.1 & 1120.5 & -16.6 \\
\hline 50 & 139 & 1142.0 & 1121.9 & -20.1 \\
\hline 50 & 140 & 1146.7 & 1125.3 & -21.4 \\
\hline
\end{tabular}

See table 5 for the estimated and total binding energies of $\mathrm{N}=2 \mathrm{Z}$ nuclides starting from $\mathrm{Z}=20$ to 50 .

Table 5: Binding energy of $\mathrm{N}=2 \mathrm{Z}$ nuclides

\begin{tabular}{|c|c|c|c|c|}
\hline $\begin{array}{c}\text { Proton } \\
\text { number }\end{array}$ & $\begin{array}{c}\text { Mass } \\
\text { number }\end{array}$ & $\begin{array}{c}\text { Est. BE } \\
(\mathrm{Mev})\end{array}$ & $\begin{array}{c}\text { Exp. } \\
\mathrm{BE}(\mathrm{Mev}) \\
{[40,42]}\end{array}$ & $\begin{array}{c}\text { Error } \\
(\mathrm{MeV})\end{array}$ \\
\hline 20 & 40 & 344.6 & 342.1 & -2.6 \\
\hline 22 & 44 & 380.8 & 375.5 & -5.3 \\
\hline 24 & 48 & 415.3 & 411.5 & -3.8 \\
\hline 26 & 52 & 450.7 & 447.7 & -3.0 \\
\hline 28 & 56 & 484.2 & 484.0 & -0.3 \\
\hline 30 & 60 & 517.3 & 515.0 & -2.3 \\
\hline 32 & 64 & 551.6 & 546.0 & -5.6 \\
\hline 34 & 68 & 583.8 & 576.3 & -7.5 \\
\hline 36 & 72 & 615.5 & 606.9 & -8.6 \\
\hline 38 & 76 & 646.8 & 638.1 & -8.7 \\
\hline 40 & 80 & 677.6 & 668.4 & -9.2 \\
\hline 42 & 84 & 707.9 & 700.9 & -7.0 \\
\hline 44 & 88 & 737.8 & 731.4 & -6.4 \\
\hline 46 & 92 & 767.3 & 762.1 & -5.2 \\
\hline 48 & 96 & 793.9 & 793.4 & -0.5 \\
\hline 50 & 100 & 822.4 & 824.5 & 2.1 \\
\hline
\end{tabular}


A large nuclear gravitational constant and its universal applications

Figure 3: Binding energy of isotopes of $\mathrm{Z}=22$

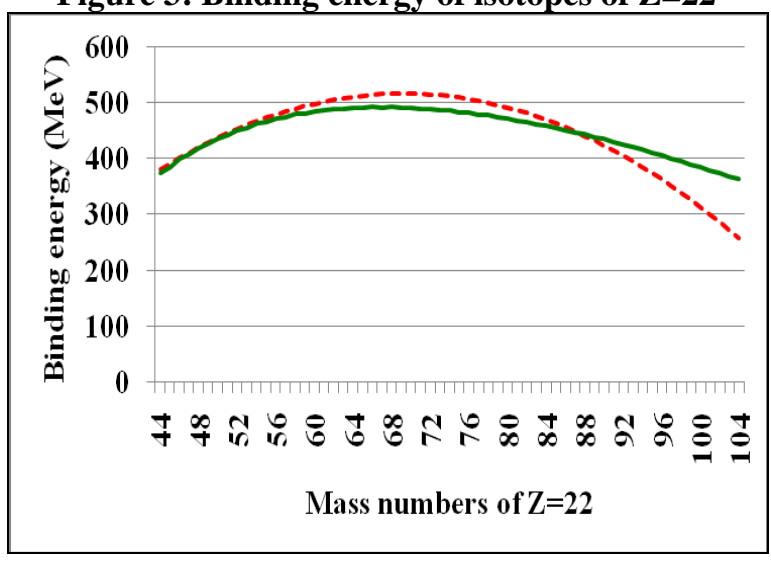

Figure 4: Binding energy of isotopes of $\mathrm{Z}=32$

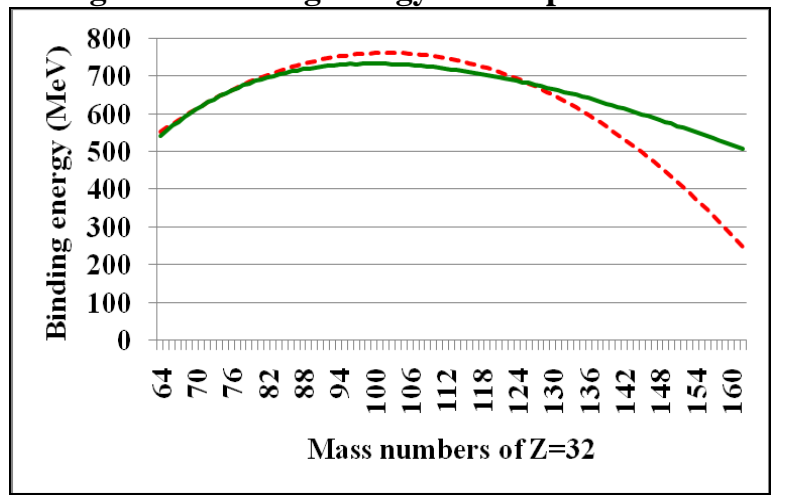

Figure 5: Binding energy of isotopes of $Z=42$

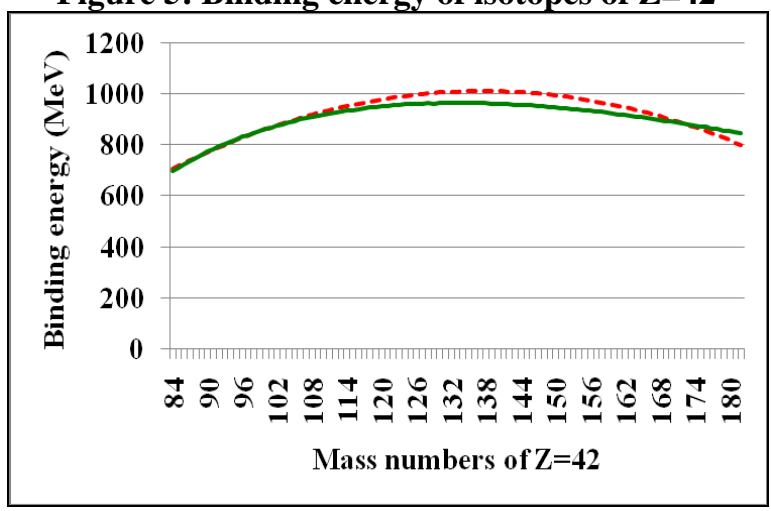

Figure 6: Binding energy of isotopes of $Z=52$

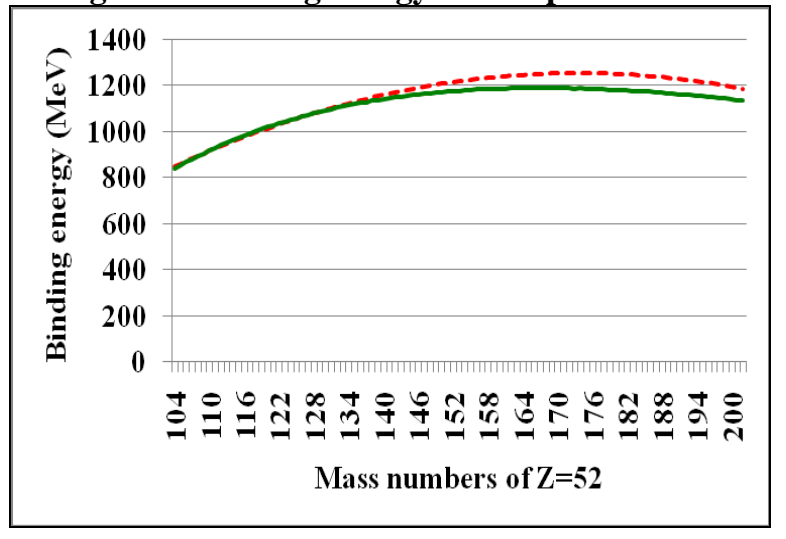

Figure 7: Binding energy of isotopes of $Z=62$

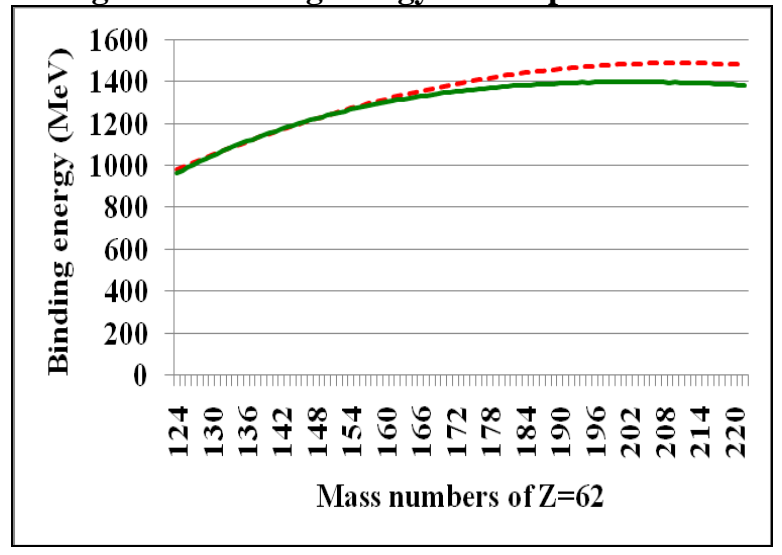

Figure 8: Binding energy of isotopes of $Z=72$

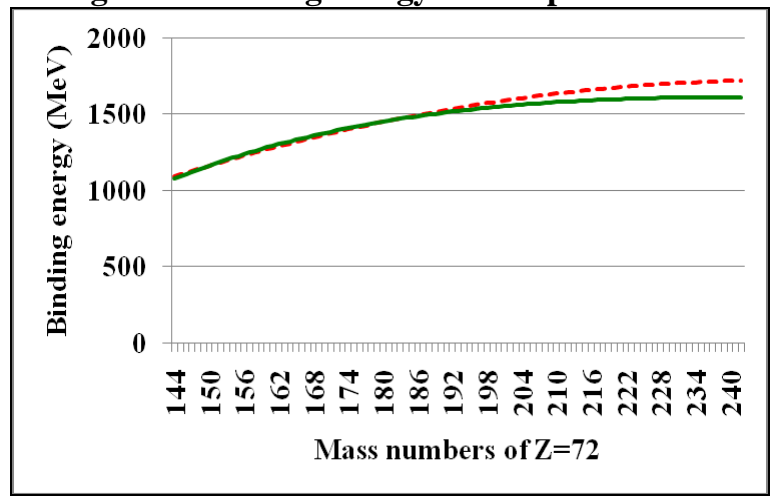

Figure 9: Binding energy of isotopes of $\mathrm{Z}=82$

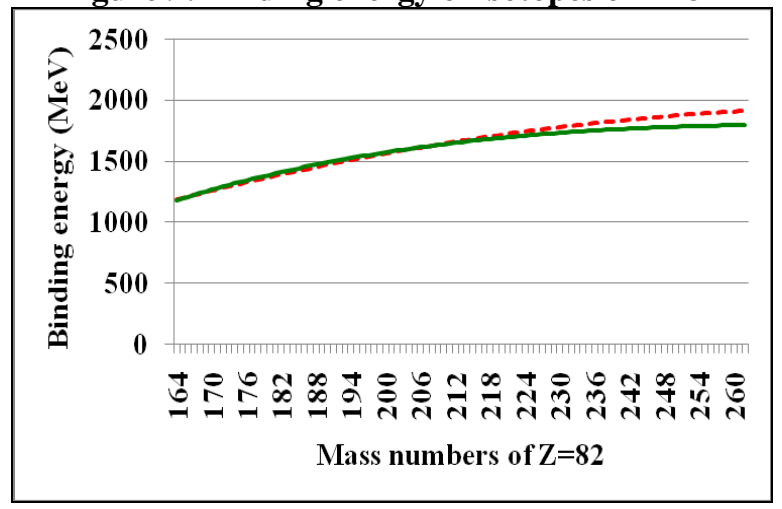

Figure 10: Binding energy of isotopes of $Z=92$

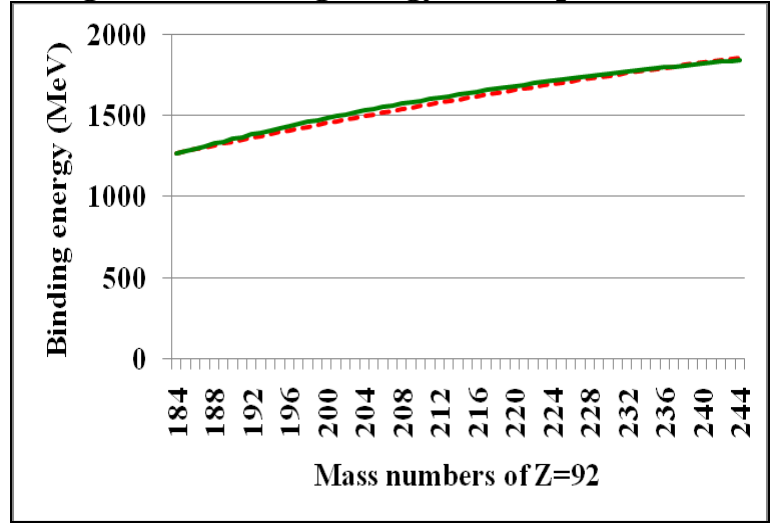


A large nuclear gravitational constant and its universal applications

\section{Understanding the binding energy of light} atomic nuclides

It is well established that, in light atomic nuclides, coulombic interaction seems to play a key role in reducing the binding energy. Based on this concept, starting from $Z=2$ to $Z=30$, close to stable mass numbers, binding energy can be expressed with the following relation.

$$
\begin{aligned}
B_{A_{s}} & \cong\left[A_{s}-A_{s}^{\frac{1}{3}}\right](10.06-0.71) \mathrm{MeV} \\
& \cong\left[A_{s}-A_{s}^{\frac{1}{3}}\right] 9.35 \mathrm{MeV}
\end{aligned}
$$

See the following table 6 .

Table 6: Binding energy of $Z=2$ to 30 based on

\begin{tabular}{|c|c|c|c|c|}
\hline $\begin{array}{l}\text { Proton } \\
\text { number }\end{array}$ & $\begin{array}{c}\text { Mass } \\
\text { number }\end{array}$ & $\begin{array}{c}\text { Est. BE } \\
(\mathrm{Mev})\end{array}$ & $\begin{array}{l}\text { SEMF BE } \\
(\mathrm{Mev}) \text { [38] }\end{array}$ & $\begin{array}{l}\text { Error } \\
(\mathrm{MeV})\end{array}$ \\
\hline 2 & 4 & 22.6 & 22.0 & -0.5 \\
\hline 3 & 6 & 39.1 & 26.9 & -12.2 \\
\hline 4 & 8 & 56.1 & 52.9 & -3.2 \\
\hline 5 & 10 & 73.4 & 62.3 & -11.1 \\
\hline 6 & 12 & 90.8 & 87.4 & -3.4 \\
\hline 7 & 14 & 108.4 & 98.8 & -9.6 \\
\hline 8 & 16 & 126.0 & 123.2 & -2.8 \\
\hline 9 & 19 & 152.7 & 148.9 & -3.8 \\
\hline 10 & 21 & 170.6 & 167.5 & -3.0 \\
\hline 11 & 23 & 188.5 & 186.1 & -2.3 \\
\hline 12 & 25 & 206.4 & 204.7 & -1.7 \\
\hline 13 & 27 & 224.4 & 223.2 & -1.2 \\
\hline 14 & 29 & 242.4 & 241.6 & -0.8 \\
\hline 15 & 31 & 260.5 & 260.0 & -0.5 \\
\hline 16 & 34 & 287.6 & 290.8 & 3.2 \\
\hline 17 & 36 & 305.7 & 305.1 & -0.7 \\
\hline 18 & 38 & 323.9 & 327.2 & 3.4 \\
\hline 19 & 40 & 342.0 & 341.5 & -0.5 \\
\hline 20 & 43 & 369.3 & 371.6 & 2.3 \\
\hline 21 & 45 & 387.5 & 389.6 & 2.1 \\
\hline 22 & 47 & 405.7 & 407.5 & 1.8 \\
\hline 23 & 49 & 423.9 & 425.2 & 1.3 \\
\hline 24 & 52 & 451.3 & 454.6 & 3.3 \\
\hline 25 & 54 & 469.6 & 468.9 & -0.7 \\
\hline 26 & 56 & 487.8 & 489.6 & 1.8 \\
\hline 27 & 59 & 515.3 & 515.2 & 0.0 \\
\hline 28 & 61 & 533.5 & 532.5 & -1.0 \\
\hline 29 & 63 & 551.8 & 549.7 & -2.2 \\
\hline 30 & 66 & 579.3 & 577.9 & -1.4 \\
\hline
\end{tabular}
coulombic correction

\section{Understanding magic proton numbers}

It may be noted that, the nuclear magic numbers, as we know in stable and naturally occurring nuclei, consist of two different series of numbers. The first series $-2,8,20$ is attributed to the harmonic-oscillator (HO) potential, while the second one - 28, 50, 82 and
126 is due to the spin-orbit ( $\mathrm{SO}$ ) coupling force [4346]. In this context, our bold idea is that, atoms are

exceptionally stable when their nuclear binding energy approaches,

$$
B_{A_{s}} \cong\left[2.531\left(n+\frac{1}{2}\right)\right]^{2} 10.06 \mathrm{MeV}
$$

Based on point 5 of section-3, close to stable mass numbers of $\mathrm{Z} \approx(2$ to 100$)$, magnitude of nuclear binding energy can be expressed with a relation of following form.

$$
\begin{aligned}
& B_{A_{s}} \approx\left\{(Z-\sqrt{\ln (Z)}) \frac{e_{s}^{2}}{4 \pi \varepsilon_{0}\left(G_{s} m_{p} / c^{2}\right)}\right\} \pm 10.06 \mathrm{MeV} \\
& \approx[(Z-\sqrt{\ln (Z)}) * 20.12 \mathrm{MeV}] \pm 10.06 \mathrm{MeV} \\
& \text { where } A_{s} \approx 2 Z+0.0063326(Z)^{2}
\end{aligned}
$$

See the following figure 11 for the plotted (dotted) black curve compared with SEMF green curve.

\section{Figure 11: Nuclear Binding energy close to stable} mass numbers of $Z=2$ to 100

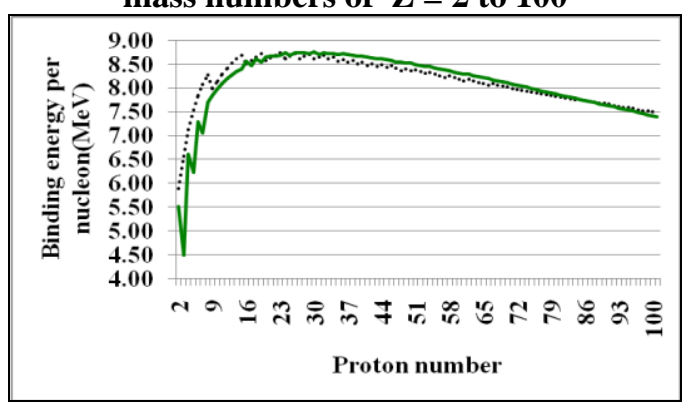

Let $M_{n}$ be a possible magic proton number. Considering relations (23) and (24), it is possible to develop a relation of the following form having a factor $(1 / 2)$.

$$
\begin{aligned}
M_{n} \cong\left\{\frac{1}{2}\left[2.531\left(n+\frac{1}{2}\right)\right]^{2}+1\right\}+\Delta \\
\cong\left[3.203\left(n+\frac{1}{2}\right)^{2}+1\right]+\Delta
\end{aligned}
$$

where, after rounding off,

$$
\begin{aligned}
& \text { if, }\left\{\frac{1}{2}\left[2.531\left(n+\frac{1}{2}\right)\right]^{2}+1\right\} \text { is Odd, } \Delta=\mp 1 \\
& \text { if, }\left\{\frac{1}{2}\left[2.531\left(n+\frac{1}{2}\right)\right]^{2}+1\right\} \text { is Even, } \Delta=\mp 2
\end{aligned}
$$

See the following table-7. It is possible to say that, 
A large nuclear gravitational constant and its universal applications

1) Magic proton numbers 2 , (6), (14), 28, 50, 82, $114, .$. etc [44-46] can be shown to be $n^{\text {th }}$ levels.

2) Magic proton numbers 2, 8, 20, 40,.. can be shown to be $\left(n+\frac{1}{2}\right)$ levels.

Table-7: To understand the magic proton numbers

\begin{tabular}{|c|c|c|}
\hline$\left(n+\frac{1}{2}\right)$ & {$\left[3.203\left(n+\frac{1}{2}\right)^{2}+1\right]$} & $M_{n}$ \\
\hline 0 & 1 & 1,2 \\
\hline 0.5 & 2 & 2,4 \\
\hline 1 & 4 & $2,4,6$ \\
\hline 1.5 & 8 & $6,8,10$ \\
\hline 2 & 14 & $12,14,16$ \\
\hline 2.5 & 21 & $20,21,22$ \\
\hline 3 & 30 & $28,30,32$ \\
\hline 3.5 & 40 & $38,40,42$ \\
\hline 4 & 52 & $50,52,54$ \\
\hline 4.5 & 66 & $64,66,68$ \\
\hline 5 & 81 & $80,81,82$ \\
\hline 5.5 & 98 & $96,98,100$ \\
\hline 6 & 116 & $114,116,118$ \\
\hline 6.5 & 136 & $134,136,138$ \\
\hline 7 & 158 & $156,158,160$ \\
\hline 7.5 & 181 & $180,181,182$ \\
\hline 8 & 206 & $204,206,208$ \\
\hline
\end{tabular}

\section{Discussion}

1) With reference to the proposed characteristic mass unit of $\sqrt{\hbar c / G_{s}} \cong 546.62 \mathrm{MeV} / c^{2}$, basic baryonic mass spectrum can be fitted with the following relation,

$m_{B a r} c^{2} \cong\left(\frac{n}{\alpha_{s}}\right)^{\frac{1}{4}} \sqrt{\frac{\hbar c^{5}}{G_{s}}} \cong\left(\frac{n}{\alpha_{s}}\right)^{\frac{1}{4}} 546.6 \mathrm{MeV}$

where $n=1,2,3, \ldots$

See table-8. For further details, readers are encouraged to see our published paper [33].

Table-8: Estimated basic baryons rest energy

\begin{tabular}{|c|c|c|c|}
\hline$n$ & $\begin{array}{c}\text { Baryon } \\
\text { rest energy } \\
(\mathrm{MeV})\end{array}$ & $n$ & $\begin{array}{c}\text { Baryon } \\
\text { rest energy } \\
(\mathrm{MeV})\end{array}$ \\
\hline 1 & 938.3 & 11 & 1708.7 \\
\hline 2 & 1115.8 & 12 & 1746.3 \\
\hline 3 & 1234.8 & 13 & 1781.6 \\
\hline 4 & 1326.9 & 14 & 1814.9 \\
\hline 5 & 1403.0 & 15 & 1846.5 \\
\hline 6 & 1468.5 & 16 & 1876.5 \\
\hline 7 & 1526.1 & 17 & 1905.2 \\
\hline
\end{tabular}

\begin{tabular}{|c|c|c|c|}
8 & 1578.0 & 18 & 1932.6 \\
\hline 9 & 1625.1 & 19 & 1958.9 \\
\hline 10 & 1668.5 & 20 & 1984.2 \\
\hline
\end{tabular}

Considering, $\left(\frac{n(n+1)}{\alpha_{s}}\right)^{\frac{1}{4}}$ or $\left(\frac{n(n+1)}{2 \alpha_{s}}\right)^{\frac{1}{4}}$ other simple baryons can also be fitted.

2) So far no model could succeed in understanding nuclear binding energy with gravity [19]. It can be confirmed from main stream literature [1-20].

3) So far no model could address or succeed in implementing strong coupling constant in low energy nuclear physics.

4) So far no model could attempt to understand nuclear stability and binding energy with the combined effects of strong nuclear gravity and strong nuclear charge.

5) Understanding nuclear binding energy with a single energy coefficient of magnitude $\frac{e_{s}^{2}}{8 \pi \varepsilon_{0}\left(G_{s} m_{p} / c^{2}\right)} \cong 10.09 \mathrm{MeV}$ is a challenging task and so far, except Ghahramany et al, no one could attempt to do that. It may also be noted that, in Ghahramany's model, energy constant is a variable [47] and in our model energy constant remains same for any nuclide.

6) Estimation of nucleon stability range is simple in our model compared to SEMF and Ghahramany's model. Interesting point to be noted is that, in our model, nucleon stability range or stable mass numbers can be estimated without considering the binding energy formula. We have provided different relations for understanding nucleon stability.

7) Proposed new and result oriented number $k \cong\left(\frac{4 \pi \varepsilon_{0} \hbar^{2} m_{e} c^{2}}{4 e^{2} G_{s} m_{p}^{3}}\right) \cong 0.0063326$ seems to play a key role in understanding nuclear stability and binding energy vide relations (6), (7), (8), (9), (10), (16) and (20).

8) Proposed first tem is not new and proposed second

$\left[\left(k A_{s} Z / 2.531\right)+3.531\right] \times 10.06 \mathrm{MeV}$ seems to

play an excellent role in fitting and understanding the binding energy of medium and heavy stable nuclides. It can be evidenced form table-3. Correction seems to be required for light atomic nuclides. It needs further study.

9) Proposed third term $\left[\left(A_{s}-A\right)^{2} / A_{s}\right] \times 10.06 \mathrm{MeV}$ seems to be approximate in fitting and understanding the binding energy of isotopes. We are working on it 
A large nuclear gravitational constant and its universal applications

for its validity and better alternative with respect correct stable mass number of $\mathrm{Z}$. For example, see the following table-9.

Table 9: Binding energy of isotopes of $\mathrm{Z}=\mathbf{8 ,}, \mathbf{1 0}$ and 20

\begin{tabular}{|c|c|c|c|c|}
\hline $\begin{array}{l}\text { Proton } \\
\text { number }\end{array}$ & $\begin{array}{c}\text { Mass } \\
\text { number }\end{array}$ & $\begin{array}{c}\text { Est. BE } \\
(\mathrm{Mev})\end{array}$ & $\begin{array}{c}\text { Total BE } \\
(\mathrm{Mev})\end{array}$ & $\begin{array}{c}\text { Error } \\
(\mathrm{MeV})\end{array}$ \\
\hline 8 & 14 & 100.0 & 98.7352 & -1.25 \\
\hline 8 & 15 & 111.7 & 111.9576 & 0.23 \\
\hline 8 & 16 & 122.2 & 127.6211 & 5.40 \\
\hline 8 & 17 & 131.4 & 131.7646 & 0.32 \\
\hline 8 & 18 & 139.4 & 139.8091 & 0.39 \\
\hline 8 & 19 & 146.1 & 143.7665 & -2.37 \\
\hline 10 & 17 & 123.6 & 112.9107 & -10.64 \\
\hline 10 & 18 & 136.7 & 132.1432 & -4.57 \\
\hline 10 & 19 & 148.9 & 143.7827 & -5.14 \\
\hline 10 & 20 & 160.2 & 160.6521 & 0.49 \\
\hline 10 & 21 & 170.5 & 167.4136 & -3.04 \\
\hline 10 & 22 & 179.8 & 177.7751 & -2.01 \\
\hline 10 & 23 & 188.2 & 182.9756 & -5.18 \\
\hline 10 & 24 & 195.6 & 191.841 & -3.72 \\
\hline 20 & 36 & 297.1 & 281.3644 & -15.69 \\
\hline 20 & 37 & 309.6 & 296.1548 & -13.50 \\
\hline 20 & 38 & 321.8 & 313.1263 & -8.65 \\
\hline 20 & 39 & 333.4 & 326.4138 & -7.03 \\
\hline 20 & 40 & 344.6 & 342.0563 & -2.58 \\
\hline 20 & 41 & 355.4 & 350.4187 & -4.94 \\
\hline 20 & 42 & 365.6 & 361.9002 & -3.72 \\
\hline 20 & 43 & 375.4 & 369.8327 & -5.58 \\
\hline 20 & 44 & 384.7 & 380.9652 & -3.77 \\
\hline 20 & 45 & 393.6 & 388.3797 & -5.21 \\
\hline 20 & 46 & 402.0 & 398.7791 & -3.20 \\
\hline 20 & 47 & 409.9 & 406.0556 & -3.84 \\
\hline 20 & 48 & 417.3 & 415.9961 & -1.35 \\
\hline 20 & 49 & 424.3 & 421.1426 & -3.19 \\
\hline 20 & 50 & 430.8 & 427.495 & -3.35 \\
\hline
\end{tabular}

10) In deuteron, binding energy seems to be proportional to $e^{2}$ and in other atomic nuclides, binding energy seems to be proportional to $e_{s}^{2}$.

11) Considering the average of $\left(e^{2}, e_{s}^{2}\right)$ and without considering $0.71 \mathrm{MeV}$ (as there exists only one proton), based on relation (22), binding energies of ${ }_{1}^{2} \mathrm{H}$ and ${ }_{1}^{3} \mathrm{H}$ nuclides can be estimated as, $\left[2-2^{\frac{1}{3}}\right] 5.6 \cong 4.15 \mathrm{MeV}$
$\left[3-3^{\frac{1}{3}}\right] 5.6 \cong 8.72 \mathrm{MeV}$ respectively.

12) Considering the average of $\left(e^{2}, e_{s}^{2}\right)$ and considering $0.71 \mathrm{MeV}$ (since there exists two protons), based on relation (22), binding energy of ${ }_{2}^{3} \mathrm{He}$ can be estimated as, $\left[3-3^{\frac{1}{3}}\right] 4.9 \cong 7.63$ $\mathrm{MeV}$.
13) Coulombic energy coefficient being $0.7 \mathrm{MeV}$, with reference to $\ln \left(\frac{e^{2}}{4 \pi \varepsilon_{0} G_{s} m_{p} m_{e}}\right) \cong 1.515$, volume or surface energy coefficient can be expressed as $1.515^{*} 10.09=15.3 \mathrm{MeV}$ and asymmetric energy coefficient can be expressed as, $1.515 * 15.3=23.0 \mathrm{MeV}$. For $(\mathrm{Z} \geq 10)$, binding energy can also be estimated with,

$$
\begin{aligned}
& B_{A} \cong\left(A-A^{2 / 3}-1\right) * 15.3 \mathrm{MeV} \\
& -\frac{Z^{2}}{A^{1 / 3}} * 0.7 \mathrm{MeV}-\frac{(A-2 Z)^{2}}{A} * 23.0 \mathrm{MeV} \\
& \text { where, }\left\{\begin{array}{l}
\frac{15.3 \mathrm{MeV}}{10.09 \mathrm{MeV}} \cong \frac{23.0 \mathrm{MeV}}{15.3 \mathrm{MeV}} \\
\cong \ln \left(\frac{e^{2}}{4 \pi \varepsilon_{0} G_{s} m_{p} m_{e}}\right) \cong 1.515
\end{array}\right.
\end{aligned}
$$

14) With advanced research in high energy nuclear physics, hadronic melting points can be understood and bare quarks can be made identifiable.

15) With further research in nuclear astrophysics, it is certainly possible to understand the combined effects of Newtonian gravitational constant and proposed nuclear gravitational constant. Considering the ratio of nuclear gravitational constant and Newtonian gravitational constant, estimated masses of white dwarfs, neutron stars and black holes [48,49], can be fitted approximately. For example,

$$
\left.\begin{array}{l}
M_{X} \approx\left(\frac{G_{s}}{G_{N}}\right) \sqrt{\frac{e^{2}}{4 \pi \varepsilon_{0} G_{N}}} \approx 0.473 M_{\square} \\
M_{X} \approx\left(\frac{G_{s}}{G_{N}}\right) \sqrt{\frac{e_{s}^{2}}{4 \pi \varepsilon_{0} G_{N}}} \approx 1.373 M_{\square} \\
M_{X} \approx\left(\frac{G_{s}}{G_{N}}\right) \sqrt{\frac{\hbar c}{G_{N}}} \approx 5.456 M_{\square}
\end{array}\right\}
$$

16) At the moment of a neutron star's birth, the nucleons that compose it have a temperature of 
around $10^{11}$ to $10^{12} \mathrm{~K}$ [50]. Equating a black hole's mass-energy density and thermal energy density $[51,52,53]$, it is possible to show that,

$$
\begin{gathered}
T_{B} \cong 0.4615 \frac{\hbar c^{3}}{k_{B} G_{N} \sqrt{M_{B} M_{p l}}} . \\
\text { where, } M_{p l} \cong \sqrt{\frac{\hbar c}{G_{N}}} \cong 2.176 \times 10^{-8} \mathrm{~kg} \\
M_{B} \cong \text { Mass of blackhole }
\end{gathered}
$$

and $T_{B} \cong$ Temperature of blackhole

This just resembles famous Hawking's Black hole temperature formula [54] with a change in its effective mass $\sqrt{M_{B} M_{p l}}$. With reference to relation (29), considering $M_{X}$ as a critical mass for neutron stars and black holes, corresponding critical temperature can be fitted with,

$$
T_{X} \approx \frac{\hbar c^{3}}{8 \pi k_{B} G_{N} \sqrt{M_{B} M_{p l}}}
$$

17) Quantitatively, Fermi's weak coupling constant [55] and electron rest mass can be fitted with the following relations.

$$
\begin{array}{r}
G_{F} \cong\left(\frac{m_{e}}{m_{p}}\right)^{2} \hbar c R_{0}^{2} \cong \frac{4 G_{s}^{2} m_{e}^{2} \hbar}{c^{3}} \\
m_{e} \cong \sqrt{\frac{G_{F} c^{3}}{4 G_{s}^{2} \hbar}} \text { and } \frac{2 G_{s} m_{e}}{c^{2}} \cong \sqrt{\frac{G_{F}}{\hbar c}}
\end{array}
$$

18) In a theoretical and verifiable approach, magnitude of the Newtonian gravitational constant can be estimated with nuclear elementary physical constants [56, 57]. For example, with reference to Planck scale, we noticed that,

$$
\frac{\pi R_{0}^{2}}{\pi R_{p l}^{2}} \cong \frac{G_{s}^{2} m_{p}^{2}}{G_{N} \hbar c} \cong\left(\frac{m_{p}}{m_{e}}\right)^{12}
$$

where, $R_{0} \cong \frac{2 G_{s} m_{p}}{c^{2}}, R_{p l} \cong \frac{2 G_{N} M_{p l}}{c^{2}} \cong 2 \sqrt{\frac{G_{N} \hbar}{c^{3}}}$

$$
\left(\frac{m_{p}}{m_{e}}\right) \cong\left(\frac{G_{s} m_{p}^{2}}{\hbar c} \times \frac{G_{s}}{G_{N}}\right)^{\frac{1}{12}} \cong\left(\frac{e_{s} G_{s}}{e G_{N}}\right)^{\frac{1}{12}}
$$

$$
\begin{gathered}
G_{N} \cong\left(\frac{m_{e}}{m_{p}}\right)^{10}\left(\frac{G_{F} c^{2}}{4 \hbar^{2}}\right) \cong\left(\frac{m_{e}}{m_{p}}\right)^{12}\left(\frac{G_{s} m_{p}^{2}}{\hbar c}\right) G_{s} \\
G_{F} \cong\left(\frac{m_{p}}{m_{e}}\right)^{10} \frac{4 \hbar^{2} G_{N}}{c^{2}} \\
\left(\frac{m_{p}}{m_{e}}\right) \cong\left(\frac{G_{F} c^{2}}{4 \hbar^{2} G_{N}}\right)^{\frac{1}{10}}
\end{gathered}
$$

19) Another very interesting relation is,

$$
\left(\frac{m_{p}}{m_{e}}\right)^{10} \cong \exp \left(\frac{1}{\alpha_{s}}\right)^{2}
$$

20) If, $G_{s} \cong \frac{4 \pi \varepsilon_{0} h^{2} c^{2} m_{e}}{e^{2} m_{p}^{3}} \cong 3.329561 \times 10^{28} \frac{\mathrm{m}^{3}}{\mathrm{~kg} \cdot \mathrm{sec}^{2}}$

$$
\left\{\begin{array}{l}
\alpha_{s} \cong 0.115194 \\
G_{F} \cong 1.44021 \times 10^{-62} \mathrm{~J} . \mathrm{m}^{3} \\
G_{N} \cong 6.679856 \times 10^{-11} \mathrm{~m}^{3} \mathrm{~kg}^{-1} \mathrm{sec}^{-2}
\end{array}\right\}
$$

21) If $\alpha_{s} \cong\left[\sqrt{\ln \left(\frac{m_{p}}{m_{e}}\right)^{10}}\right]^{-1} \cong 0.1153515$,

$$
\left\{\begin{array}{l}
G_{s} \cong 3.327283 \times 10^{28} \mathrm{~m}^{3} \mathrm{~kg}^{-1} \mathrm{sec}^{-2} \\
G_{F} \cong 1.43824 \times 10^{-62} \mathrm{~J} \cdot \mathrm{m}^{3} \\
G_{N} \cong 6.670719 \times 10^{-11} \mathrm{~m}^{3} \mathrm{~kg}^{-1} \mathrm{sec}^{-2}
\end{array}\right\}
$$

22) With reference to the macroscopic Planck's constant and microscopic strong coupling constant, average values seem to be:

$$
\left\{\begin{array}{l}
\alpha_{s} \cong 0.115273 \\
G_{s} \cong 3.32842 \times 10^{28} \mathrm{~m}^{3} \mathrm{~kg}^{-1} \mathrm{sec}^{-2} \\
G_{F} \cong 1.43922 \times 10^{-62} \mathrm{~J} \cdot \mathrm{m}^{3} \\
G_{N} \cong 6.675285 \times 10^{-11} \mathrm{~m}^{3} \mathrm{~kg}^{-1} \mathrm{sec}^{-2}
\end{array}\right\}
$$

23) Relations (34), (35) and (38) seem to indicate the direct role of $G_{N}$ in microscopic physics. We are working on understanding their physical significance with respect to proton-electron mass ratio.

24) Our proposed assumptions seem to ease the way of understanding and refining the basic concepts of final unification $[58,59,60]$. 
A large nuclear gravitational constant and its universal applications

\section{Conclusion}

Liquid drop model, Fermi gas model, quantum chromodynamics and string theory models are lagging in implementing the strong coupling constant and gravity in basic nuclear structure. In this context, understanding and estimating nuclear binding energy with 'strong interaction' and 'unification' concepts seem to be quite interesting and needs a serious consideration at basic level. Even though they are semi empirical, section (3) and relations (6), (7), (8), (9), (10), (11), (20), (21), (24), (26), (27), (28), (29), (30), (31), (34), (35), (38), (39) and (42) can be considered as favorable or supporting tools for our proposed model. One very interesting point to be noted is that, our proposed model seems to SPAN across the Fermi scale and Planck scale. With further research, mystery of magic numbers can be understood and a unified model of nuclear binding energy and stability scheme pertaining to high and low energy nuclear physics can be developed.

\section{Acknowledgements}

Authors are very much thankful to Dr. N. Ghahramany and team for their intuitive and heuristic contributions in this most advanced field of nuclear research. Author Seshavatharam is indebted to professors shri M. Nagaphani Sarma, Chairman, shri K.V. Krishna Murthy, founder Chairman, Institute of Scientific Research in Vedas (I-SERVE), Hyderabad, India and Shri K.V.R.S. Murthy, former scientist IICT (CSIR), Govt. of India, Director, Research and Development, I-SERVE, for their valuable guidance and great support in developing this subject.

\section{References}

[1] K. Tennakone. (1974). Electron, muon, proton, and strong gravity. Phys. Rev. D 10, 1722

[2] Perng J. J. Strong gravitation and elementary particles. Nuovo Cimento, Lettere, Serie 2, vol. 23, N. 15, pp. $552-554$ (1978).

[3] Salam, Abdus; Sivaram, C. (1993). Strong Gravity Approach to QCD and Confinement. Modern Physics Letters A, 8 (4): 321-326.

[4] Salam, A., and Strathdee, J. (1978). Confinement Through Tensor Gauge Fields. Physical Review D 18, 4596-4609

[5] Sivaram, C, Sinha, K. (1977). Strong gravity, black holes, and hadrons. Physical Review D. 16 (6): 1975-1978.

[6] Sivaram C and Sinha K.P. Strong spin-two interactions and general relativity. Phys. Rep., 1979, v. 51, 113-187 (p.152)

[7] C. Sivaram et al. (2013). Gravity of Accelerations on Quantum Scales. Preprint, arXiv:1402.5071

[8] Recami E. and Tonin-Zanchin V. The strong coupling constant: its theoretical derivation from a geometric approach to hadron structure. Found. Phys. Lett., 1994, v, 7(1), 85-92

[9] Recami E. et al. The strong coupling constant. APH N.S., Heavy Ion Physics 10 (1999) 345-349

[10] Raut, Usha and Shina, KP. Strong gravity and the fine structure constant. Proceedings of the Indian Academy of Sciences Part A: Physical Sciences, 49 (2). pp. 352-358.

[11] V. de Sabbata and C. Sivaram. Strong SpinTorsion Interaction between Spinning Protons. IL Nuovo Cimento, Vol. 101A, N. 2, pp. 273-283 (1989)

[12] Roberto Onofrio. (2013). Proton radius puzzle and quantum gravity at the Fermi scale. EPL 104, 20002

[13] O. F. Akinto, Farida Tahir. (2017) Strong Gravity Approach to QCD and General Relativity. arXiv:1606.06963v3

[14] T. R. Mongan. Dark matter from "strong gravity" - consistent with CRESST, CoGeNT and DAMA/LIBRA. arXiv:0706.3050v3, Dec 2011

[15] J. Dufour. Very sizeable increase of gravity at pico-meter distance: a novel working hypothesis to explain anomalous heat effects and apparent transmutations in certain metal hydrogen systems. J. of condensed matter nuclear science, Vol. 1, pp. 47-61 (2007).

[16] Oldershaw R.L. Discrete Scale Relativity. Astrophysics and Space Science, Vol. 311, N. 4, pp. 431-433 (2007).

[17] Oldershaw R.L. Hadrons As Kerr-Newman Black Holes, Journal of Cosmology, Vol. 6, 1361-1374,2010

[18] Stone R.A. Quark Confinement and Force Unification. Progress in Physics, April 2010, Vol. 2, P. 19-20.

[19] Sergey G. Fedosin. The radius of the proton in the self-consistent model. Hadronic Journal, Vol. 35, No. 4, pp. 349-363 (2012).

[20] S. I. Fisenko, M. M. Beilinson and B. G. Umanov. Some notes on the concept of "strong" gravitation and possibilities of its experimental investigation. Physics Letters A, Volume 148, Issues 8-9, pp. 405-407 (1990).

[21] Seshavatharam U.V.S \& Lakshminarayana S.. Strong nuclear gravitational constant and the origin of nuclear planck scale. Progress in Physics, vol. 3, pp. 31-38 (2010).

[22] Seshavatharam U.V.S \& Lakshminarayana S, On the role of strong interaction in understanding nuclear beta stability line and nuclear binding energy. Proceedings of the DAE-BRNS Symp. On Nucl. Phys. 60, 118-119 (2015)

[23] Seshavatharam U.V.S \& Lakshminarayana S, On the role of 'reciprocal' of the strong coupling constant in nuclear structure. To be appeared in 
A large nuclear gravitational constant and its universal applications

Journal of Nuclear Sciences, Ankara University, Turkey.

[24] Seshavatharam U.V.S \& Lakshminarayana S, Understanding Nuclear Stability and Binding Energy with Very Large Gravitational coupling and Strong Nuclear Charge. To be appeared in the proceedings of ICNPAP conference, October, 2018, Centre for Applied Physics, Central University of Jharkhand, Ranchi, India.

[25] Seshavatharam U.V.S \& Lakshminarayana S, On the possible existence of strong elementary charge and its applications. To be appeared in the proceedings of ICNPAP conference, October, 2018, Centre for Applied Physics, Central University of Jharkhand, Ranchi, India. Seshavatharam

[26] Seshavatharam U.V.S \& Lakshminarayana S, A new approach to understand nuclear stability and binding energy. Proceedings of the DAE-BRNS Symp. On Nucl. Phys. 62, 106-107 (2017)

[27] Seshavatharam U.V.S \& Lakshminarayana S, Understanding the constructional features of materialistic atoms in the light of strong nuclear gravitational coupling. Materials Today: 3/10PB, Proceedings 3 (2016) pp. 3976-3981

[28] Seshavatharam U.V.S \& Lakshminarayana S, Understanding the basics of final unification with three gravitational constants associated with nuclear, electromagnetic and gravitational interactions. Journal of Nuclear Physics, Material Sciences, Radiation and Applications Vol-4, No1, 1-19, (2017)

[29] Seshavatharam U.V.S \& Lakshminarayana S, A Virtual Model of Microscopic Quantum Gravity. Prespacetime Journal, Vol. 9, Issue 1, pp. 58-82 (2018)

[30] Seshavatharam U.V.S \& Lakshminarayana S, (2015). To confirm the existence of nuclear gravitational constant, Open Science Journal of Modern Physics. 2(5): 89-102

[31] Seshavatharam U.V.S \& Lakshminarayana S, (2016) Towards a workable model of final unification. International Journal of Mathematics and Physics 7, No1,117-130.

[32] Seshavatharam U.V.S \& Lakshminarayana S, (2015) Lakshminarayana. To Validate the Role of Electromagnetic and Strong Gravitational Constants via the Strong Elementary Charge. Universal Journal of Physics and Application 9(5): 210-219

[33] Seshavatharam U.V.S \& Lakshminarayana S. Scale Independent Workable Model of Final Unification. Universal Journal of Physics and Application 10(6): 198-206, 2016.
[34] Seshavatharam U.V.S \& Lakshminarayana S, To unite nuclear and sub-nuclear strong interactions. International Journal of Physical Research, 5 (2) 104-108 (2017)

[35] Seshavatharam U.V.S \& Lakshminarayana S, On the role of strong coupling constant and nucleons in understanding nuclear stability and binding energy. Journal of Nuclear Sciences, Vol. 4, No.1, 7-18, (2017)

[36] Seshavatharam U.V.S \& Lakshminarayana S, A Review on Nuclear Binding Energy Connected with Strong Interaction. Prespacetime Journal, Vol. 8, Issue 10, pp. 1255-1271 (2018)

[37] Seshavatharam U.V.S \& Lakshminarayana S, Simplified Form of the Semi-empirical Mass Formula. Prespacetime Journal, Volume 8, Issue 7, pp.881-810 (2017)

[38] Chowdhury, P.R. et al. Modified BetheWeizsacker mass formula with isotonic shift and new driplines. Mod. Phys. Lett. A20 p.16051618. (2005).

[39] Oganessian, Yu \& K Utyonkov, V. (2015). Super-heavy element research. Reports on progress in physics. Physical Society (Great Britain). 78. 036301.

[40] Ghahramany $\mathrm{N}$ et al. New approach to nuclear binding energy in integrated nuclear model. Physics of Particles and Nuclei Letters, 2011, Vol. 8, No. 2, pp. 97-106.

[41] Ghahramany $\mathrm{N}$ et al. New scheme of nuclide and nuclear binding energy from quark-like model. Iranian Journal of Science \& Technology (2011) A3: 201-208

[42] W. D. Myers et al. Table of Nuclear Masses according to the 1994 Thomas-Fermi Model.(from nsdssd.lbl.gov)

[43] Seshavatharam U.V.S \& Lakshminarayana S. On the Possible Existence of Strong Elementary Charge \& Its Applications. Prespacetime Journal, Vol. 9, Issue 7, pp. 642-651 (2018)

[44] Ghahramany N et al. Quark-Gluon Plasma Model and the Origin of Magic Numbers. Iranian Physical Journal, 1-2, 35-38 (2007).

[45] Tran, D. T. et al, Evidence for prevalent $Z=6$ magic number in neutron-rich carbon isotopes. Nature Communications, Vol 9, Article number: 1594 (2018)

[46] Fridmann J, et al. Magic nucleus 42Si. Nature. 2005; 435:922-924.

[47] Ghahramany N et al. Stability and Mass Parabola in Integrated Nuclear Model. Universal Journal of Physics and Application 1(1): 18-25, 2013. 
[48] Ludwig, Hendrik \& Ruffini, Remo. (2014). Gamow's Calculation of the Neutron Star Critical Mass Revised. Journal of the Korean Physical Society. 65. 10.3938/jkps.65.892.

[49] I.F. Mirabel. The formation of stellar black holes. New Astronomy Reviews Volume 78, August 2017, 1-15

[50] https://en.wikipedia.org/wiki/Neutron_star

[51] Seshavatharam U.V.S \& Lakshminarayana S. Nuclear mass density based high temperature stable black holes. Proceedings of the DAE Symp. on Nucl. Phys. 59, 804-805 (2014)

[52] Seshavatharam U.V.S \& Lakshminarayana S. Primordial Hot Evolving Black Holes and the Evolved Primordial Cold Black Hole Universe. Frontiers of Astronomy, Astrophysics and Cosmology, 2015, Vol. 1, No. 1, 16-23.

[53] Mitra A. Non-occurrence of Trapped Surfaces and Black Holes in Spherical Gravitational Collapse. Foundations of Physics Letters.13:543579. (2000)

[54] S. Hawking S.W. Particle creation by black holes. Commun. Math. Phys. 43: 199-220. (1975)

[55] C. Patrignani et al. (Particle Data Group), Chin. Phys. C, 40, 100001 (2016) and 2017 update

[56] Jonathan L. Feng et al. Probing Gravitational Interactions of Elementary Particles. International Journal of Modern Physics DVol. 13, No. 10, pp. 2355-2359 (2004)

[57] Brandenburg J. E. The GEM Unification Theory of the Vacuum: Did Dimensional Collapse Trigger the Big Bang. International Journal of Astrophysics and Space Science. Special Issue: Quantum Vacuum, Fundamental Arena of the Universe: Models, Applications and Perspectives. Vol. 2, No. 6-1, pp. 24-38. (2014).

[58] S. Bethke and G.P. Salam. Quantum chromodynamics. K.A. Olive et al. (Particle Data Group), Chin. Phys. C, 38, 090001 (2014) and 2015 update.

[59] K. Becker, M. Becker and J. H. Schwarz. String Theory and M-theory: A Modern Introduction. Cambridge University Press, (2006)

[60] https://aeon.co/essays/has-the-quest-for-topdown-unification-of-physics-stalled 